\title{
Detector modules and spectrometers for the TIME-Pilot [CII] intensity mapping experiment
}

Jonathon Hunacek, James Bock, C. Matt Bradford, Bruce Bumble, Tzu-Ching Chang, et al.

Jonathon Hunacek, James Bock, C. Matt Bradford, Bruce Bumble, TzuChing Chang, Yun-Ting Cheng, Asantha Cooray, Abigail Crites, Steven Hailey-Dunsheath, Yan Gong, Chao-Te Li, Roger O'Brient, Erik Shirokoff, Corwin Shiu, Jason Sun, Zachary Staniszewski, Bade Uzgil, Michael Zemcov, "Detector modules and spectrometers for the TIME-Pilot [CII] intensity mapping experiment," Proc. SPIE 9914, Millimeter, Submillimeter, and Far-Infrared Detectors and Instrumentation for Astronomy VIII, 99140L (19 July 2016); doi: 10.1117/12.2233762

Event: SPIE Astronomical Telescopes + Instrumentation, 2016, Edinburgh, United Kingdom 


\title{
Detector Modules and Spectrometers for the TIME-Pilot [CII] Intensity Mapping Experiment
}

\author{
Jonathon Hunacek ${ }^{\mathrm{a}}$, James Bock ${ }^{\mathrm{a}, \mathrm{b}}$, C. Matt Bradford ${ }^{\mathrm{b}, \mathrm{a}}$, Bruce Bumble ${ }^{\mathrm{b}}$, Tzu-Ching Chang ${ }^{\mathrm{c}}$, \\ Yun-Ting Cheng ${ }^{\mathrm{a}}$, Asantha Cooray ${ }^{\mathrm{d}}$, Abigail Crites ${ }^{\mathrm{a}}$, Steven Hailey-Dunsheath ${ }^{\mathrm{a}}$, Yan Gong', \\ Chao-Te Li ${ }^{\mathrm{c}}$, Roger O'Brient ${ }^{\mathrm{b}, \mathrm{a}}$, Erik Shirokoff ${ }^{\mathrm{f}}$, Corwin Shiu ${ }^{\mathrm{a}}$, Jason Sun ${ }^{\mathrm{a}}$, Zachary Staniszewski ${ }^{\mathrm{b}}$, \\ Bade Uzgil ${ }^{\mathrm{d}}$, and Michael Zemcov ${ }^{\mathrm{e}}$ \\ ${ }^{a}$ California Institute of Technology, Pasadena, USA \\ ${ }^{\mathrm{b}}$ Jet Propulsion Laboratory, Pasadena, USA \\ ${ }^{c}$ Academia Sinica Institute of Astronomy and Astrophysics, Taipei, Taiwan \\ ${ }^{\mathrm{d}}$ University of California Irvine, Irvine, USA \\ ${ }^{\mathrm{e}}$ Rochester Institute of Technology, Rochester, USA \\ ${ }^{\mathrm{f}}$ University of Chicago, Chicago, USA

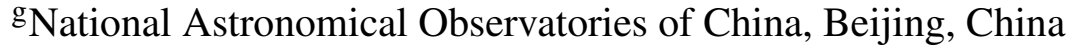

\begin{abstract}
This proceeding presents the current TIME-Pilot instrument design and status with a focus on the close-packed modular detector arrays and spectrometers. Results of laboratory tests with prototype detectors and spectrometers are discussed.

TIME-Pilot is a new mm-wavelength grating spectrometer array under development that will study the Epoch of Reionization (the period of time when the first stars and galaxies ionized the intergalactic medium) by mapping the fluctuations of the redshifted $157.7 \mu \mathrm{m}$ emission line of singly ionized carbon ([CII]) from redshift $z \sim 5.2$ to 8.5. As a tracer of star formation, the [CII] power spectrum can provide information on the sources driving reionization and complements $21 \mathrm{~cm}$ data (which traces neutral hydrogen in the intergalactic medium). Intensity mapping provides a measure of the mean [CII] intensity without the need to resolve and detect faint sources individually. We plan to target a 1 degree by 0.35 arcminute field on the sky and a spectral range of $199-305 \mathrm{GHz}$, producing a spatial-spectral slab which is $140 \mathrm{Mpc}$ by $0.9 \mathrm{Mpc}$ on-end and $1230 \mathrm{Mpc}$ in the redshift direction. With careful removal of intermediate-redshift CO sources, we anticipate a detection of the halo-halo clustering term in the [CII] power spectrum consistent with current models for star formation history in 240 hours on the JCMT.

TIME-Pilot will use two stacks of 16 parallel-plate waveguide spectrometers (one stack per polarization) with a resolving power $R \sim 100$ and a spectral range of 183 to $326 \mathrm{GHz}$. The range is divided into 60 spectral channels, of which 16 at the band edges on each spectrometer serve as atmospheric monitors. The diffraction gratings are curved to produce a compact instrument, each focusing the diffracted light onto an output arc sampled by the 60 bolometers. The bolometers are built in buttable dies of 8 (low freqeuency) or 12 (high frequency) spectral channels by 8 spatial channels and are mated to the spectrometer stacks. Each detector consists of a gold micro-mesh absorber and a titanium transition edge sensor (TES). The detectors (1920 total) are designed to operate from a $250 \mathrm{mK}$ base temperature in an existing cryostat with a photon-noise-dominated NEP of $\sim 2 * 10^{-17} \mathrm{~W} \mathrm{~Hz}^{-1 / 2}$. A set of flexible superconducting cables connect the detectors to a time-domain multiplexing SQUID readout system.
\end{abstract}

Keywords: reionization, intensity mapping, [CII], spectrometers, bolometers, transition edge sensors

\section{SCIENTIFIC MOTIVATION}

The early universe was a hot, dense plasma which, by a redshift $z \sim 1100$, had expanded and cooled to the point where it could condense into neutral hydrogen in a processed referred to as recombination. In the proceeding "Dark Ages," this neutral hydrogen collapsed into matter overdensities, forming early stars and galaxies. These first luminous objects produced lyman-contiuum phontons capable of reionizing the neutral hydrogen in the intergalactic medium, thus forming ionized bubbles surrounding them. As these bubbles grew and merged, the universe transitioned to the fully reionized state we observe today. ${ }^{1}$ Understanding this reionization process requires knowledge of the timing and duration of reionization 


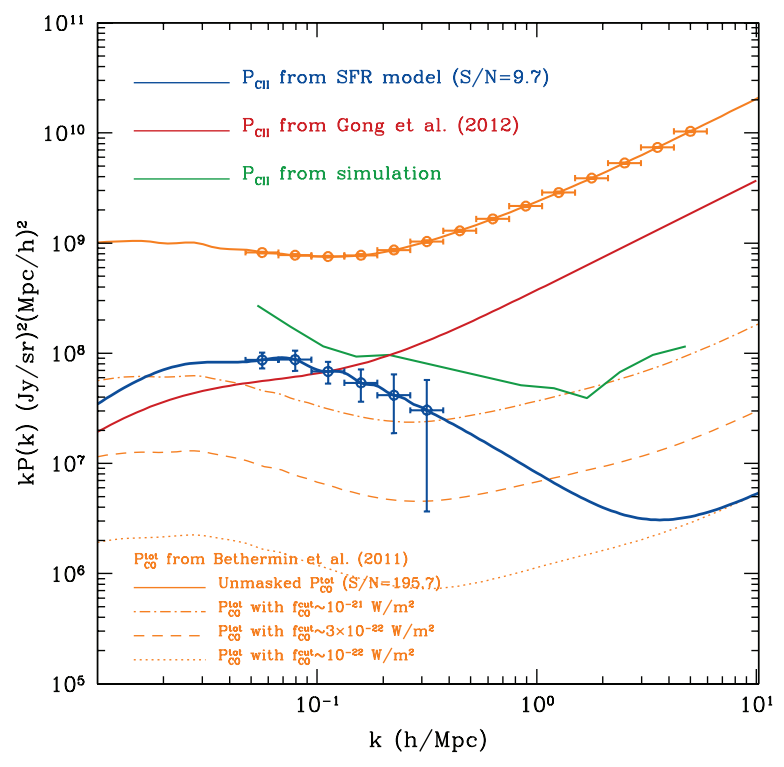

Figure 1. Three models (red, ${ }^{16}$ blue, ${ }^{16}$ green ${ }^{17}$ ) are shown for the [CII] power spectrum (in units of $k P(k)$ ) at $z \sim 7$. The expected total lower-redshift CO signal from several dominant transitions is shown in solid orange. ${ }^{18,19}$ The broken orange lines correspond to the remaining $\mathrm{CO}$ signal after masking down to different $\mathrm{CO}$ flux limits. We anticipate a detection of the clustering term of the [CII] power spectrum with a signal to noise ratio of $\sim 10$ after masking no more than $20 \%$ of our survey voxels.

as well as an understanding of the sources driving it. Direct observation of rest-frame UV emission from high redshift galaxies could answer some of these questions; however, the steep faint-end slope of the UV luminosity function at high redshift forces uncertain extrapolation beyond current measurements to compute the total number of available UV photons. ${ }^{2}$ This indicates that the bulk of the UV light produced by early reionization-era galaxies is coming from a large number of small, faint sources. Measuring many such sources remains a challenge for conventional galaxy surveys; thus, alternative probes of reionization are required. Observations of the spectra of high-redshift quasars show supression from absoption by neutral hydrogen along the line of sight, producing what is called a Gunn-Peterson Trough. ${ }^{3}$ Even a small neutral component in the intergalactic medium can produce large supression; as a result, this method is only sensitive to the end of the Epoch of Reionization, with current measurements indicating the process was complete at $z \sim 6{ }^{4-6}$ Further information can be gathered from the effects of reionization on the power spectrum of the cosmic microwave background. Free electrons in the reionized universe scatter CMB photons, producing an effective optical depth dependent on the midpoint of reionization that affects the E-mode polarization power spectrum at large angular scales $(l \lesssim 20){ }^{7}$ Additionally, the bulk motion of ionized bubbles affects the CMB temperature power spectrum at small angular scales $(l \sim 3000)$ through the kinetic Sunyaev-Zeldovich effect ${ }^{8}$ in a way that depends on the duration of reionization. ${ }^{7,9}$ The latest results from Planck (including small-scale temperature data from $\mathrm{SPT}^{9}$ and $\mathrm{ACT}^{10}$ ) find the midpoint of reionzation to be $z \sim 7.8-8.8$ with a duration $\Delta z<2.8 .^{11}$ Additionally, several projects are currently working to map the $21 \mathrm{~cm}$ emission of neutral hydrogen from the Epoch of Reionization, ${ }^{12-14}$ with recent results including an upper limit on the $21 \mathrm{~cm}$ power spectrum at $z=8.4$ by PAPER-64. ${ }^{15}$ This method shows great promise to probe the neutral medium, but foreground challenges remain.

TIME-Pilot ${ }^{20-23}$ will take advantage of the 157.7 um emission line of singly ionized carbon ([CII]), a bright cooling line which is thought to be a useful tracer star formation in high redshift galaxies. ${ }^{16,17}$ [CII] emission is redshifted into the $\mathrm{mm}$-wavelength atmospheric window from a redshift $z \sim 5.2$ to $z \sim 8.5$, permitting the use of ground-based intrumentation and allowing us to leverage exisiting CMB detector technologies. This technique is complementary to $21 \mathrm{~cm}$ studies, and cross-correlation between $[\mathrm{CII}]$ and $21 \mathrm{~cm}$ datasets will be a powerful tool in reducing foreround contamination in both measurements as well as in accessing the scale of reionization bubbles. ${ }^{16}$

TIME-Pilot will utilize a techique called intensity mapping, in which large beams integrate photons from many dim sources to produce a spatial-spectral data cube. Science results are extracted from the power spectrum of this cube (Fig. 

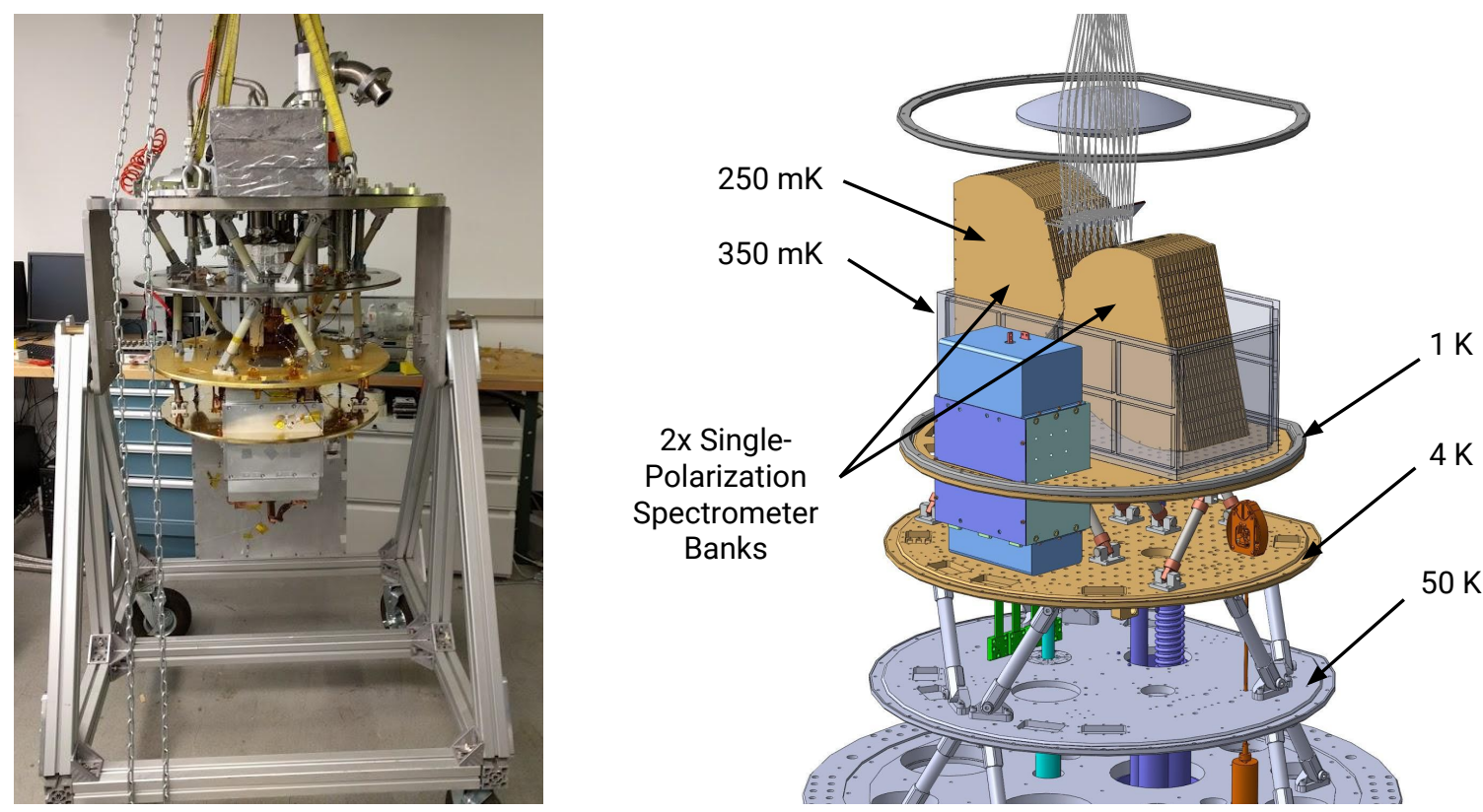

Figure 2. Left: The TIME-Pilot cryostat at Caltech. Right: The TIME-Pilot instrument showing the placement of the two spectrometer banks. Cryostat shields and full input optics are not shown.

1), analogous to the $\mathrm{CMB}$ with the addition of a third dimension. Because were are not resolving individual sources, we are sensitive to the many faint galaxies that would remain undetected in a traditional galaxy survey.

The dominant foreground challenge for [CII] mapping during the Epoch of Reionizaion will be $\mathrm{CO}$ emission from lower redshift galaxies. Because of our spectroscopic approach, we have the ability to eject voxels containing known bright $\mathrm{CO}$ emitters though the use of low redshift galaxy tracers. By masking 10-20\% of our survey voxels, we anticipate that we will supress $\mathrm{CO}$ contamination sufficiently to allow a detection of the two-halo clustering term of the [CII] power spectrum at $z \sim 7$ with a signal to noise ratio of $\sim 10$. This measurement would constrain the mean [CII] intensity (and thus the cosmic star formation rate) at that redshift. Successful component separation will also produce a rich CO data set during the peak of cosmic star formation $(z \sim 2)$. Novel foreground separation methods are under development to further address this. ${ }^{24}$

\section{INSTRUMENT OVERVIEW}

TIME-Pilot is designed to have an instanteous field of view of $11 \times 0.35$ arcminutes, which we have broken into $16 \times 1$ spatial pixels. The instrument consists of two banks of grating spectrometers, each responsible a single polarization. Each bank (Fig. 3) contains 16 grating spectrometers with a resolution $R \sim 100$ (channel widths of $\sim 2-3 \mathrm{GHz}$ ) and a spectral coverage of 183 to $326 \mathrm{GHz}$. For each spectrometer, a multi-flare-angle smooth-walled split-block feedhorn launches a single mode into a parallel plate waveguide. A curved diffraction grating, similar to that used in Z-Spec ${ }^{25,26}$ but with a lower resolving power, diffracts and focuses the light onto an output arc sampled by 60 transition edge sensor (TES) bolometers. The detectors for each bank are organzied into six blocks covering 16 pixels in the spatial direction and either 8 or 12 in the spectral direction. The two banks of spectrometers contain a total of 1920 detectors. Detector bias lines run over superconducting flex circuits to NIST SQUIDs mounted on separate readout circuit boards. We use time domain multiplexing, and data acquisition utilizes Multi-Channel Electronics (MCE) hardware from UBC. ${ }^{27}$ The TIMEPilot instrument is housed in an exisiting cryostat (Fig. 2) which uses a pulse tube refridgerator for the $50 \mathrm{~K}$ and $4 \mathrm{~K}$ stages, a continuous $4 \mathrm{He}$ Joule-Thompson fridge for the $1 \mathrm{~K}$ stage, a pair of $3 \mathrm{He}$ sorption fridges providing a continuous $350 \mathrm{mK},{ }^{28}$ and an additional $3 \mathrm{He}$ sorption ultra-cold stage to reach a base temperature of $250 \mathrm{mK}$. 


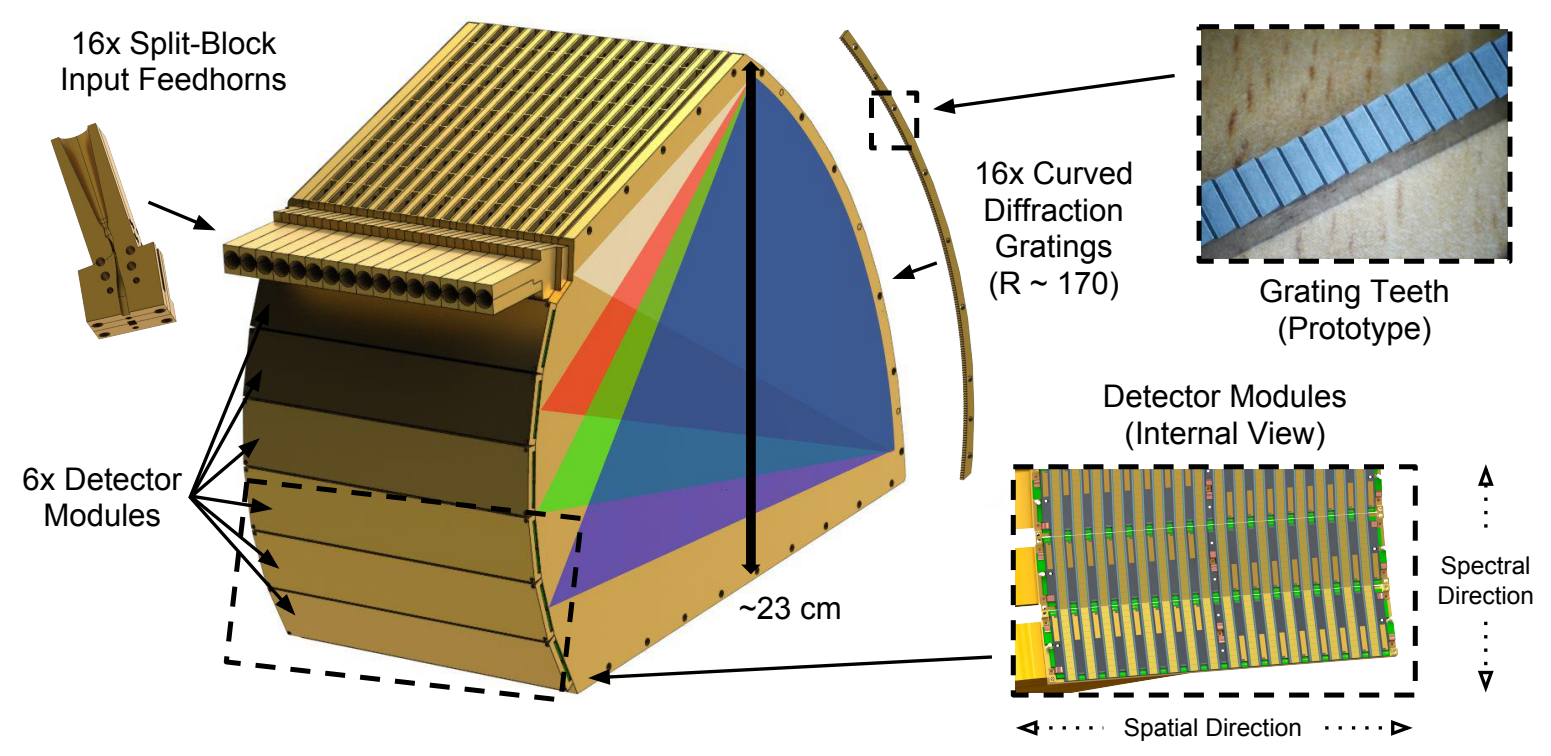

Figure 3. A single bank of 16 spectrometers, each of which consists of a split-block multi-flare-angle input feedhorn, a parallel plate waveguide, and a curved diffraction grating.

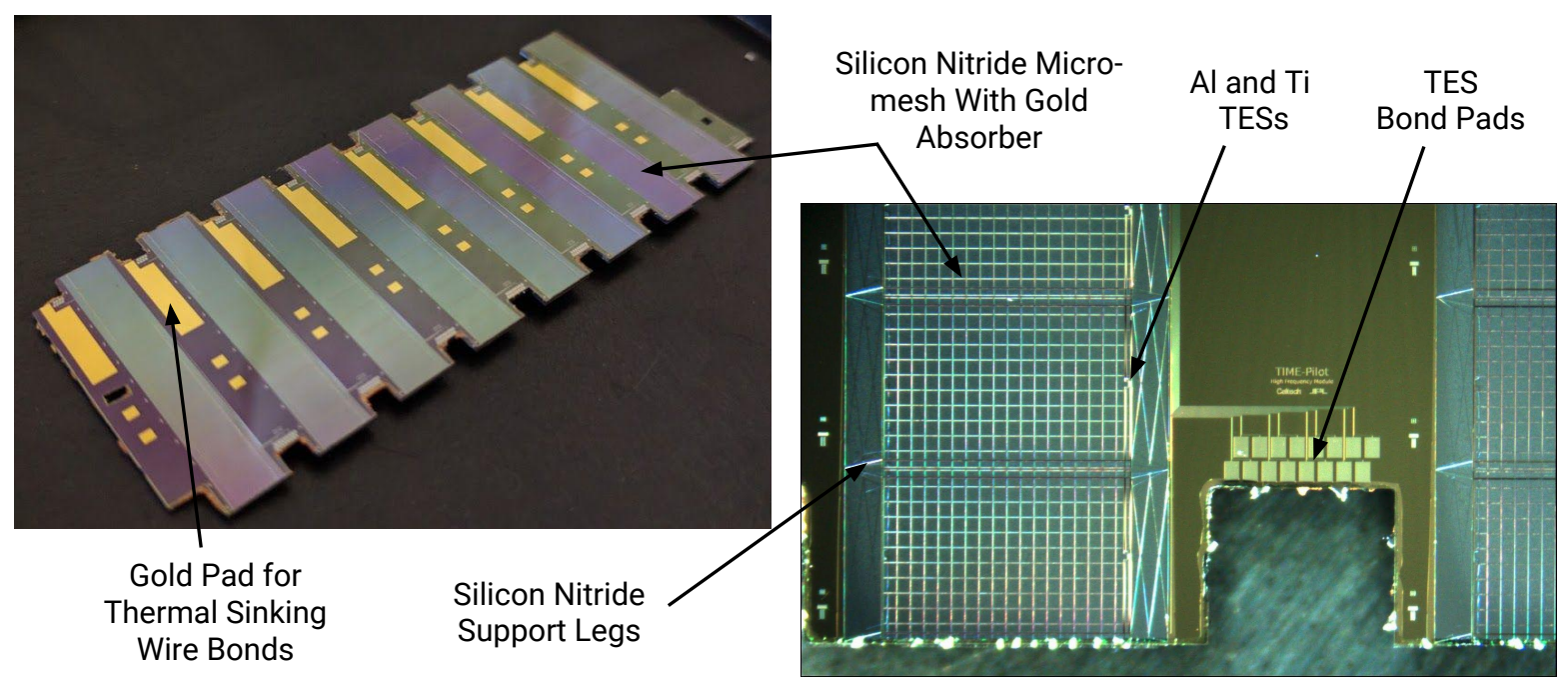

Figure 4. A prototype high-frequency detector module fabricated at JPL. Each high-frequency module is responsible for 12 spectral pixels for each of 8 spatial pixels. Similar low-frequency modules are responsible 8 spectral pixels for each of 8 spatial pixels. Each pixel consists of gold absorber on a released silicon nitride micro-mesh with aluminum and titanium TESs. 


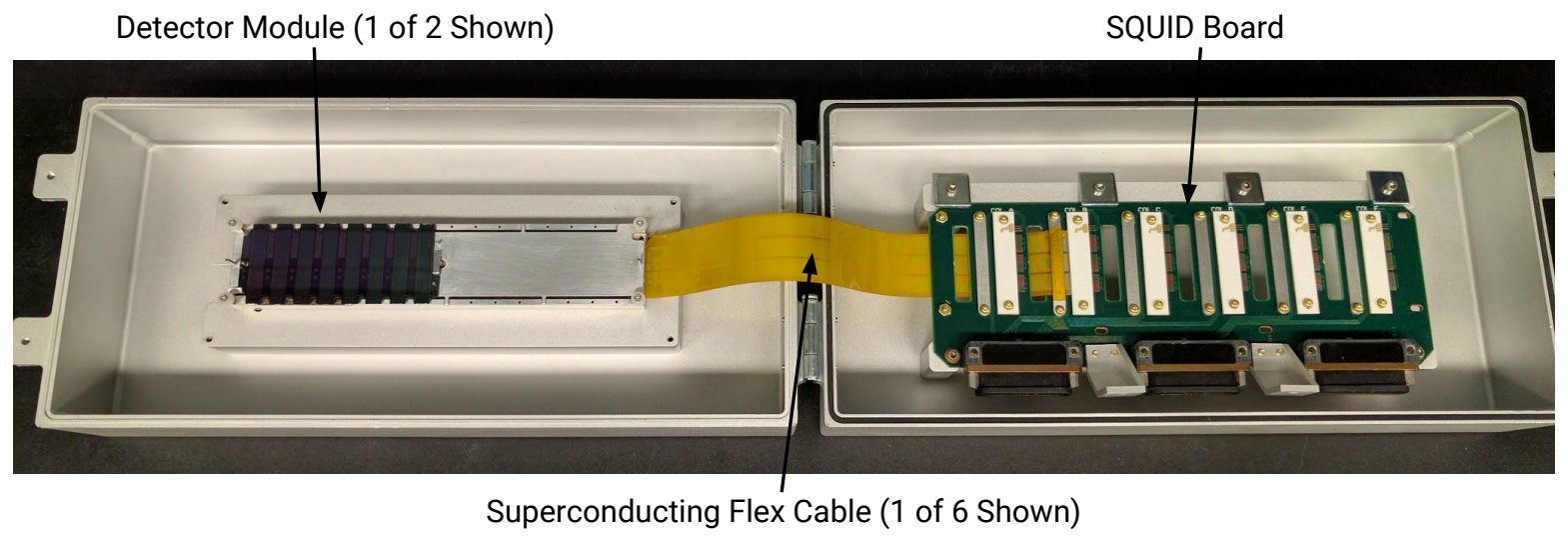

Figure 5. A partially-assembled detector block. Thin kapton flex-cables with tin-coated-copper traces carry the TES bias lines from the detector modules (left) to the readout circuitry (right). Six detector blocks mount to a single spectrometer bank.
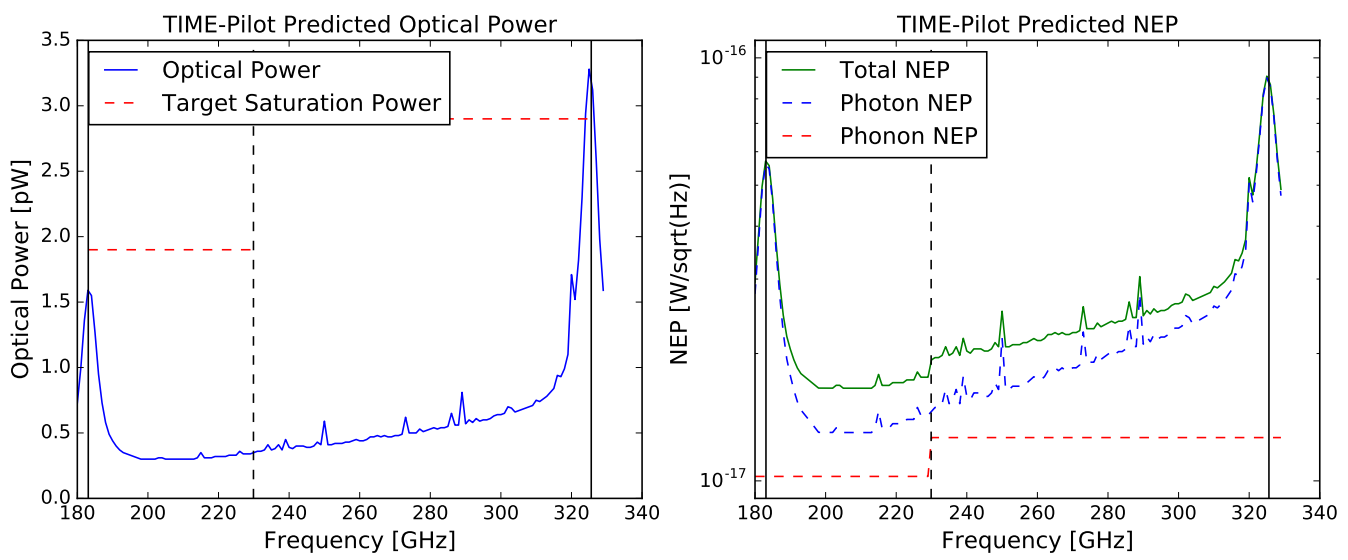

Figure 6. Left: The target detector saturation power and expected optical load for a single $R \sim 100$ channel centered on the specified frequency assuming $1 \mathrm{~mm}$ PWV at the JCMT. Right: The expected total per-detector NEP. Phonon noise is sub-dominant to photon noise. 


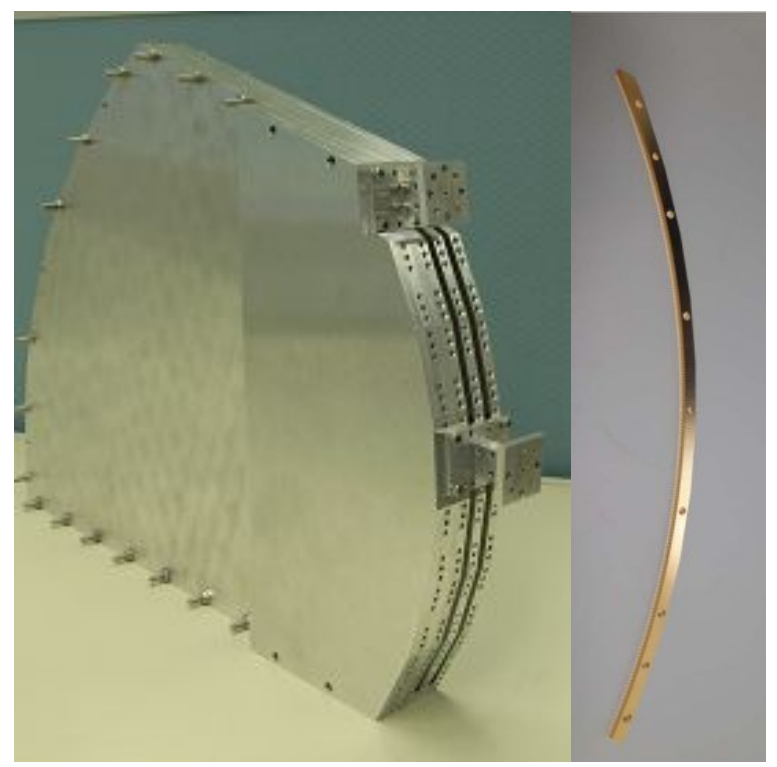

Figure 7. A prototype spectrometer bank with only two spectrometers. A full bank would consist of 16 spectrometers.

\section{DETECTOR PROTOTYPES}

Fig. 4 shows a recent TIME-Pilot high-frequency detector module prototype produced at the Micro-Devices Laboratory (MDL) at JPL. Each pixel consists of gold on a $2.3 \times 3 \mathrm{~mm}$ silicon nitride micro-mesh, which acts as a resistive sheet to absorb incident radiation. Four thin silicon nitride legs (approximately $500 \times 1 \times 1 \mu \mathrm{m}$ ) suspend the web over $10 \mu \mathrm{m}$ of vacuum and define the thermal conductance $(\mathrm{G})$ to the base temperature $(250 \mathrm{mK})$. At one side of the mesh a titatium $\left(T_{c} \sim 490 \mathrm{mK}\right)$ and an aluminum $\left(T_{c} \sim 1.2 \mathrm{~K}\right)$ TES are connected in series. During normal science loading conditions the aluminum TES is superconducting and the titanium transition is used; the aluminum transition may be used under high load laboratory conditions. The devices are fabricated on a double silicon-on-insulator (SOI) wafer, consisting of a lower $\sim 500 \mu \mathrm{m}$ silicon handle layer, a middle $\sim 80 \mu \mathrm{m}$ silicon layer defining the bulk of the quarter-wavelength backshort, and a $\sim 10 \mu \mathrm{m}$ upper silicon layer that is etched to release the silicon nitride micro-meshes. Niobium bias lines connect the TESs to wire bond pads along the perimeter of the devices. These bond pads are placed along notches (see Fig. 4) rather than being placed on the outer edge, thus leaving space for wire bonding to the circuity beneath while still allowing modules to be mounted adjacently for continuous spectral coverage (an overview of the method used to produce these notches can be found in Hunacek et. al $2016^{21}$ ). Large gold thermal pads allow heat sinking of the devices to their aluminum holders. Six superconducting flex cables (thin kapton cables with tin-coated-copper traces) route the TES bias lines to the readout circuity, as seen in Fig. 5.

As shown in Fig. 6, we are targeting a TES saturation power of $\sim 1.9 \mathrm{pW}$ for the low-frequency modules and $\sim 2.9 \mathrm{pW}$ for the high-frequency modules. This corresponds to a thermal conductance $G_{c}$ (at $T_{c}=490 \mathrm{mK}$ ) of $\sim 8 \mathrm{pW} / \mathrm{K}$ and $\sim$ $12 \mathrm{pW} / \mathrm{K}$ respectively. Detectors which achieve these targets are projected to be photon-noise dominated. Previously tested prototype devices showed an anomously high $G_{c}$; it is speculated that differential contraction at cryogenic temperatures between the deposited metals and the underlying silicon nitride resulted in physical contact between the micro-mesh and the silicon it is suspended above. The device design has been modified to mitigate this effect by keeping the mesh under sifficient tension. Testing of these improved devices is ongoing.

\section{SPECTROMETER PROTOTYPES}

A prototype bank of two spectrometers, shown in Fig. 7, has been fabricated and tested. Further details on the design of this spectrometer can be found in $\mathrm{Li}$ et. al 2016. ${ }^{29}$ A swept source was coupled to the spectrometer input, and single mode waveguide with a diode detector was placed at different fixed positions on the output arc. The resulting output profiles are shown in Fig. 8 and agree with predicted shapes. The overall predicted room-temperature efficiency is computed and compared with measurements in Table 1. Efficiencies agree with expectations to within $\sim 10 \%$. 


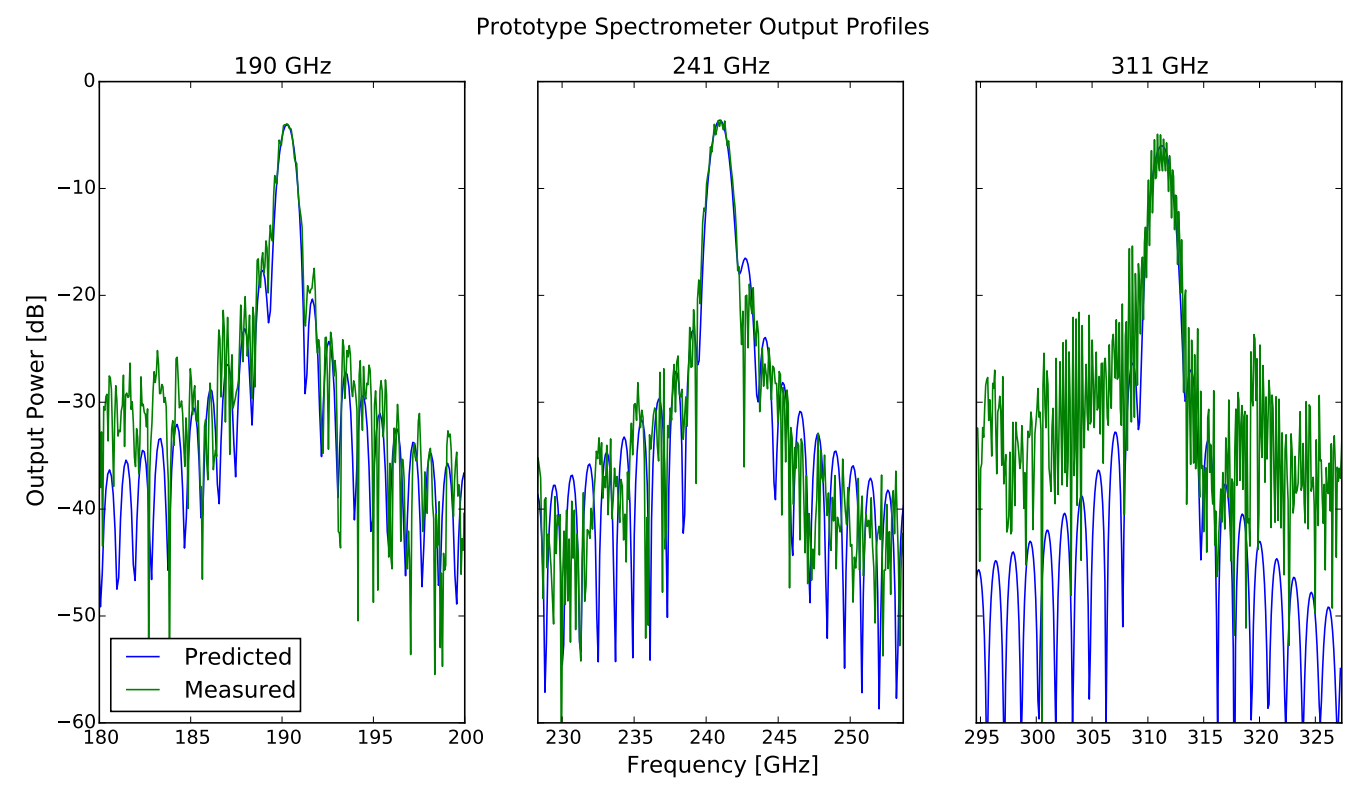

Figure 8. Measured and predicted output profiles for a prototype spectrometer bank at room temperature. The widths of the spectra shown are the convolution between the intrinsic resolution of the grating $(R>140)$ and the width of the output waveguide used for testing. The detector spacing in the final instrument is designed to provide a resolution $R \sim 100$.

\begin{tabular}{|c|c|c|c|}
\hline Frequency [GHz] & $\mathbf{1 9 0 . 3}$ & $\mathbf{2 4 1 . 0}$ & $\mathbf{3 1 1 . 3}$ \\
\hline Grating Illumination & 0.76 & 0.85 & 0.89 \\
\hline Blaze Efficiency & 0.92 & 0.97 & 0.88 \\
\hline Waveguide Propagation Efficiency & 0.97 & 0.98 & 0.99 \\
\hline Output Coupling Efficiency & 0.55 & 0.57 & 0.33 \\
\hline Predicted Total Efficiency & 0.37 & 0.46 & 0.26 \\
\hline Measured Total Efficiency & 0.40 & 0.45 & 0.25 \\
\hline Measured/Predicted Efficiency Ratio & 1.08 & 0.98 & 0.96 \\
\hline
\end{tabular}

Table 1. Measured and predicted efficiencies for a prototype spectrometer bank at room temperature. 


\section{SUMMARY AND FUTURE WORK}

We have successfully demonstrated prototype spectrometers for TIME-Pilot; fabrication of the full spectrometer banks will commence shortly. Prototype detector modules have been produced and are being characterized in a testbed at Caltech. Current modules are on track to meet design targets. Integration and testing of the full instrument is expected to be completed by late 2017 .

Upon deployment, we intend to target a 1 degree linear field, producing a spectral-spatial data slab with 156x 1 voxels in the spatial direction and 44 voxels in the spectral (redshift) direction. This line scan stategy optimizes the signal to noise ratio for a given integration time while still providing access to large scale sky modes. The TIME-Pilot survey is estimated to require 240 hours on the $15 \mathrm{~m}$ JCMT.

\section{ACKNOWLEDGMENTS}

This material is based upon work supported by the National Science Foundation Graduate Research Fellowship under Grant No. DGE-1144469.

\section{REFERENCES}

[1] Robertson, B. E., Ellis, R. S., Dunlop, J. S., McLure, R. J., and Stark, D. P., "Early star-forming galaxies and the reionization of the Universe," Nature 468, 49-55 (Nov. 2010).

[2] Livermore, R. C., Finkelstein, S. L., and Lotz, J. M., "Directly Observing the Galaxies Likely Responsible for Reionization," ArXiv e-prints (Apr. 2016).

[3] Gunn, J. E. and Peterson, B. A., "On the Density of Neutral Hydrogen in Intergalactic Space.," ApJ 142, 1633-1641 (Nov. 1965).

[4] Becker, R. H., Fan, X., White, R. L., Strauss, M. A., Narayanan, V. K., Lupton, R. H., Gunn, J. E., Annis, J., Bahcall, N. A., Brinkmann, J., Connolly, A. J., Csabai, I., Czarapata, P. C., Doi, M., Heckman, T. M., Hennessy, G. S., Ivezić, Ž., Knapp, G. R., Lamb, D. Q., McKay, T. A., Munn, J. A., Nash, T., Nichol, R., Pier, J. R., Richards, G. T., Schneider, D. P., Stoughton, C., Szalay, A. S., Thakar, A. R., and York, D. G., "Evidence for Reionization at z 6: Detection of a Gunn-Peterson Trough in a $\mathrm{z}=6.28$ Quasar," AJ 122, 2850-2857 (Dec. 2001).

[5] White, R. L., Becker, R. H., Fan, X., and Strauss, M. A., "Probing the Ionization State of the Universe at z $>6$," AJ 126, 1-14 (July 2003).

[6] Fan, X., Strauss, M. A., Becker, R. H., White, R. L., Gunn, J. E., Knapp, G. R., Richards, G. T., Schneider, D. P., Brinkmann, J., and Fukugita, M., "Constraining the Evolution of the Ionizing Background and the Epoch of Reionization with z 6 Quasars. II. A Sample of 19 Quasars,” AJ 132, 117-136 (July 2006).

[7] Reichardt, C. L., "Observing the Epoch of Reionization with the Cosmic Microwave Background," in [Astrophysics and Space Science Library], Mesinger, A., ed., Astrophysics and Space Science Library 423, 227 (2016).

[8] Sunyaev, R. A. and Zeldovich, I. B., "The velocity of clusters of galaxies relative to the microwave background - The possibility of its measurement," MNRAS 190, 413-420 (Feb. 1980).

[9] Zahn, O., Reichardt, C. L., Shaw, L., Lidz, A., Aird, K. A., Benson, B. A., Bleem, L. E., Carlstrom, J. E., Chang, C. L., Cho, H. M., Crawford, T. M., Crites, A. T., de Haan, T., Dobbs, M. A., Doré, O., Dudley, J., George, E. M., Halverson, N. W., Holder, G. P., Holzapfel, W. L., Hoover, S., Hou, Z., Hrubes, J. D., Joy, M., Keisler, R., Knox, L., Lee, A. T., Leitch, E. M., Lueker, M., Luong-Van, D., McMahon, J. J., Mehl, J., Meyer, S. S., Millea, M., Mohr, J. J., Montroy, T. E., Natoli, T., Padin, S., Plagge, T., Pryke, C., Ruhl, J. E., Schaffer, K. K., Shirokoff, E., Spieler, H. G., Staniszewski, Z., Stark, A. A., Story, K., van Engelen, A., Vanderlinde, K., Vieira, J. D., and Williamson, R., "Cosmic Microwave Background Constraints on the Duration and Timing of Reionization from the South Pole Telescope," ApJ 756, 65 (Sept. 2012).

[10] Das, S., Louis, T., Nolta, M. R., Addison, G. E., Battistelli, E. S., Bond, J. R., Calabrese, E., Crichton, D., Devlin, M. J., Dicker, S., Dunkley, J., Dünner, R., Fowler, J. W., Gralla, M., Hajian, A., Halpern, M., Hasselfield, M., Hilton, M., Hincks, A. D., Hlozek, R., Huffenberger, K. M., Hughes, J. P., Irwin, K. D., Kosowsky, A., Lupton, R. H., Marriage, T. A., Marsden, D., Menanteau, F., Moodley, K., Niemack, M. D., Page, L. A., Partridge, B., Reese, E. D., Schmitt, B. L., Sehgal, N., Sherwin, B. D., Sievers, J. L., Spergel, D. N., Staggs, S. T., Swetz, D. S., Switzer, E. R., Thornton, R., Trac, H., and Wollack, E., "The Atacama Cosmology Telescope: temperature and gravitational lensing power spectrum measurements from three seasons of data," J. Cosmology Astropart. Phys. 4, 014 (Apr. 2014). 
[11] Planck Collaboration, Adam, R., Aghanim, N., Ashdown, M., Aumont, J., Baccigalupi, C., Ballardini, M., Banday, A. J., Barreiro, R. B., Bartolo, N., Basak, S., Battye, R., Benabed, K., Bernard, J.-P., Bersanelli, M., Bielewicz, P., Bock, J. J., Bonaldi, A., Bonavera, L., Bond, J. R., Borrill, J., Bouchet, F. R., Bucher, M., Burigana, C., Calabrese, E., Cardoso, J.-F., Carron, J., Chiang, H. C., Colombo, L. P. L., Combet, C., Comis, B., Coulais, A., Crill, B. P., Curto, A., Cuttaia, F., Davis, R. J., de Bernardis, P., de Rosa, A., de Zotti, G., Delabrouille, J., Di Valentino, E., Dickinson, C., Diego, J. M., Doré, O., Douspis, M., Ducout, A., Dupac, X., Elsner, F., Enßlin, T. A., Eriksen, H. K., Falgarone, E., Fantaye, Y., Finelli, F., Forastieri, F., Frailis, M., Fraisse, A. A., Franceschi, E., Frolov, A., Galeotta, S., Galli, S., Ganga, K., Génova-Santos, R. T., Gerbino, M., Ghosh, T., González-Nuevo, J., Górski, K. M., Gruppuso, A., Gudmundsson, J. E., Hansen, F. K., Helou, G., Henrot-Versillé, S., Herranz, D., Hivon, E., Huang, Z., Ili, S., Jaffe, A. H., Jones, W. C., Keihänen, E., Keskitalo, R., Kisner, T. S., Knox, L., Krachmalnicoff, N., Kunz, M., KurkiSuonio, H., Lagache, G., Lähteenmäki, A., Lamarre, J.-M., Langer, M., Lasenby, A., Lattanzi, M., Lawrence, C. R., Le Jeune, M., Levrier, F., Lewis, A., Liguori, M., Lilje, P. B., López-Caniego, M., Ma, Y.-Z., Macías-Pérez, J. F., Maggio, G., Mangilli, A., Maris, M., Martin, P. G., Martínez-González, E., Matarrese, S., Mauri, N., McEwen, J. D., Meinhold, P. R., Melchiorri, A., Mennella, A., Migliaccio, M., Miville-Deschênes, M.-A., Molinari, D., Moneti, A., Montier, L., Morgante, G., Moss, A., Naselsky, P., Natoli, P., Oxborrow, C. A., Pagano, L., Paoletti, D., Partridge, B., Patanchon, G., Patrizii, L., Perdereau, O., Perotto, L., Pettorino, V., Piacentini, F., Plaszczynski, S., Polastri, L., Polenta, G., Puget, J.-L., Rachen, J. P., Racine, B., Reinecke, M., Remazeilles, M., Renzi, A., Rocha, G., Rossetti, M., Roudier, G., Rubiño-Martín, J. A., Ruiz-Granados, B., Salvati, L., Sandri, M., Savelainen, M., Scott, D., Sirri, G., Sunyaev, R., Suur-Uski, A.-S., Tauber, J. A., Tenti, M., Toffolatti, L., Tomasi, M., Tristram, M., Trombetti, T., Valiviita, J., Van Tent, F., Vielva, P., Villa, F., Vittorio, N., Wandelt, B. D., Wehus, I. K., White, M., Zacchei, A., and Zonca, A., "Planck intermediate results. XLVII. Planck constraints on reionization history," ArXiv e-prints (May 2016).

[12] Dillon, J. S., Neben, A. R., Hewitt, J. N., Tegmark, M., Barry, N., Beardsley, A. P., Bowman, J. D., Briggs, F., Carroll, P., de Oliveira-Costa, A., Ewall-Wice, A., Feng, L., Greenhill, L. J., Hazelton, B. J., Hernquist, L., HurleyWalker, N., Jacobs, D. C., Kim, H. S., Kittiwisit, P., Lenc, E., Line, J., Loeb, A., McKinley, B., Mitchell, D. A., Morales, M. F., Offringa, A. R., Paul, S., Pindor, B., Pober, J. C., Procopio, P., Riding, J., Sethi, S., Shankar, N. U., Subrahmanyan, R., Sullivan, I., Thyagarajan, N., Tingay, S. J., Trott, C., Wayth, R. B., Webster, R. L., Wyithe, S., Bernardi, G., Cappallo, R. J., Deshpande, A. A., Johnston-Hollitt, M., Kaplan, D. L., Lonsdale, C. J., McWhirter, S. R., Morgan, E., Oberoi, D., Ord, S. M., Prabu, T., Srivani, K. S., Williams, A., and Williams, C. L., "Empirical covariance modeling for $21 \mathrm{~cm}$ power spectrum estimation: A method demonstration and new limits from early Murchison Widefield Array 128-tile data," Phys. Rev. D 91, 123011 (June 2015).

[13] Paciga, G., Albert, J. G., Bandura, K., Chang, T.-C., Gupta, Y., Hirata, C., Odegova, J., Pen, U.-L., Peterson, J. B., Roy, J., Shaw, J. R., Sigurdson, K., and Voytek, T., "A simulation-calibrated limit on the H I power spectrum from the GMRT Epoch of Reionization experiment,” MNRAS 433, 639-647 (July 2013).

[14] Liu, A. and Parsons, A. R., "Constraining cosmology and ionization history with combined $21 \mathrm{~cm}$ power spectrum and global signal measurements," MNRAS 457, 1864-1877 (Apr. 2016).

[15] Ali, Z. S., Parsons, A. R., Zheng, H., Pober, J. C., Liu, A., Aguirre, J. E., Bradley, R. F., Bernardi, G., Carilli, C. L., Cheng, C., DeBoer, D. R., Dexter, M. R., Grobbelaar, J., Horrell, J., Jacobs, D. C., Klima, P., MacMahon, D. H. E., Maree, M., Moore, D. F., Razavi, N., Stefan, I. I., Walbrugh, W. P., and Walker, A., "PAPER-64 Constraints on Reionization: The $21 \mathrm{~cm}$ Power Spectrum at $\mathrm{z}=8.4$," ApJ 809, 61 (Aug. 2015).

[16] Gong, Y., Cooray, A., Silva, M., Santos, M. G., Bock, J., Bradford, C. M., and Zemcov, M., "Intensity Mapping of the [C II] Fine Structure Line during the Epoch of Reionization," ApJ 745, 49 (Jan. 2012).

[17] Silva, M., Santos, M. G., Cooray, A., and Gong, Y., "Prospects for Detecting C II Emission during the Epoch of Reionization,” ApJ 806, 209 (June 2015).

[18] Béthermin, M., Dole, H., Lagache, G., Le Borgne, D., and Penin, A., "Modeling the evolution of infrared galaxies: a parametric backward evolution model," A\&A 529, A4 (May 2011).

[19] Carilli, C. L. and Walter, F., “Cool Gas in High-Redshift Galaxies,” ARA\&A 51, 105-161 (Aug. 2013).

[20] Bradford, M., Crites, A., Hunacek, J., Hailey-Dunsheath, S., Zemcov, M. B., Bock, J., Cooray, A. R., Chang, T.-C., Li, C.-T., Bumble, B., Shirokoff, E., Staniszewski, Z., O’Brient, R., Shiu, C., Cheng, Y.-T., and Uzgil, B., "Probing the Epoch of Reionization via CII Tomography with TIME-Pilot," in [American Astronomical Society Meeting Abstracts ], American Astronomical Society Meeting Abstracts 227, 426.07 (Jan. 2016). 
[21] Hunacek, J., Bock, J., Bradford, C. M., Bumble, B., Chang, T.-C., Cheng, Y.-T., Cooray, A., Crites, A., HaileyDunsheath, S., Gong, Y., Kenyon, M., Koch, P., Li, C.-T., O’Brient, R., Shirokoff, E., Shiu, C., Staniszewski, Z., Uzgil, B., and Zemcov, M., "Design and fabrication of tes detector modules for the time-pilot [cii] intensity mapping experiment," Journal of Low Temperature Physics 184(3), 733-738 (2016).

[22] Crites, A. T., Bock, J. J., Bradford, C. M., Chang, T. C., Cooray, A. R., Duband, L., Gong, Y., Hailey-Dunsheath, S., Hunacek, J., Koch, P. M., Li, C. T., O’Brient, R. C., Prouve, T., Shirokoff, E., Silva, M. B., Staniszewski, Z., Uzgil, B., and Zemcov, M., "The TIME-Pilot intensity mapping experiment," in [Millimeter, Submillimeter, and Far-Infrared Detectors and Instrumentation for Astronomy VII], Proc. SPIE 9153, 91531W (Aug. 2014).

[23] Staniszewski, Z., Bock, J. J., Bradford, C. M., Brevik, J., Cooray, A., Gong, Y., Hailey-Dunsheath, S., O’Brient, R., Santos, M., Shirokoff, E., Silva, M., and Zemcov, M., "The tomographic ionized-carbon mapping experiment (time) cii imaging spectrometer," Journal of Low Temperature Physics 176(5), 767-772 (2014).

[24] Cheng, Y.-T., Chang, T.-C., Bock, J., Bradford, C. M., and Cooray, A., "Spectral Line De-confusion in an Intensity Mapping Survey," ArXiv e-prints (Apr. 2016).

[25] Bradford, C. M., Naylor, B. J., Zmuidzinas, J., Bock, J. J., Gromke, J., Nguyen, H., Dragovan, M., Yun, M., Earle, L., Glenn, J., Matsuhara, H., Ade, P. A. R., and Duband, L., "WaFIRS: a waveguide far-IR spectrometer: enabling spectroscopy of high-z galaxies in the far-IR and submillimeter," in [IR Space Telescopes and Instruments], Mather, J. C., ed., Proc. SPIE 4850, 1137-1148 (Mar. 2003).

[26] Bradford, C. M., Aguirre, J. E., Aikin, R., Bock, J. J., Earle, L., Glenn, J., Inami, H., Maloney, P. R., Matsuhara, H., Naylor, B. J., Nguyen, H. T., and Zmuidzinas, J., "The Warm Molecular Gas around the Cloverleaf Quasar,” ApJ 705, 112-122 (Nov. 2009).

[27] Battistelli, E. S., Amiri, M., Burger, B., Halpern, M., Knotek, S., Ellis, M., Gao, X., Kelly, D., MacIntosh, M., Irwin, K., and Reintsema, C., "Functional description of read-out electronics for time-domain multiplexed bolometers for millimeter and sub-millimeter astronomy," Journal of Low Temperature Physics 151(3), 908-914 (2008).

[28] Prouve, T., Duband, L., Hodis, J., Bock, J., Bradford, C. M., and Holmes, W., "Spica/bliss cryo-chain demonstrator," Cryogenics 70, 70 - 75 (2015).

[29] Li, C.-T., Wei, T., Cheng, J.-C., Shiu, C., Crites, A. T., and Bradford, C. M., "Development of a millimeter wave grating spectrometer for TIME Pilot," The 27th International Symposium on Space Terahertz Technology (2016). 